\title{
Contaminazioni
}

\author{
Maurizio Fea
}

\section{Storie edificanti dall'altro mondo}

Antonio da Padova non è il santo patrono degli enologi - quella distinzione va a San Vincenzo o San Martino di Tours o, se vi capita di essere in Bulgaria, a San Trifon il Potatore - ma forse dovrebbe esserlo, almeno nel Sud California.

Perché quando Santo Cambianica venne a Los Angeles dalla Lombardia e fondò la Cantina di San Antonio, fu la sua devozione a quel santo e alla sua chiesa che avrebbe salvato il business. Come la maggior parte dei suoi compatrioti, Cambianica era cattolico molto devoto e così chiamò la sua cantina Sant'Antonio, il santo patrono non dei produttori di vino ma delle cose perdute, dei viaggiatori, dei poveri.

Se Cambianica era un viaggiatore, non rimase tale.

Né finì povero e perduto, come fecero molti dei suoi colleghi viticoltori, quando nel 1920 il proibizionismo scosse l'industria del vino come una pesante brocca che batte su un tavolo da pranzo.

Cambianica emigrò nel centro di Los Angeles nel 1914, facendo la sua casa e iniziando la sua cantina su mezzo ettaro di terra in quella che allora era Little Italy, una fiorente rete di migliaia di immigrati dell'Europa occidentale.

Era quindi una delle più grandi concentrazioni di italo-americani ad ovest del Mississippi.

Gli italiani si stabilirono a Lincoln Heights e in quella che ora è Chinatown, venendo qui in ragione di una fiorente industria agricola e della Southern Pacific Railroad.

Era una buona posizione per un'azienda vinicola, perché c'erano vigneti nelle valli vicine, una ferrovia per trasportare il prodotto - la Red Car Line correva appena fuori le porte della cantina - e un sacco di immigrati abituati a bere il vino.

Il proibizionismo cambiò la fiorente industria vinicola californiana in un settore improvvisamente in crisi, tenuto insieme con filo spinato, barili di legno di quercia e feritoie.

II Volstead Act, che applicava il diciottesimo emendamento, esentava I'alcol usato per scopi medicinali o cosmetici, le acque da toletta e, per scopi religiosi, il vino sacramentale.

Quando la San Antonio Winery fu fondata nel 1917, tre anni prima del Proibizionismo, era una delle circa 90 aziende vinicole di Los Angeles; quando il Proibizionismo fu abrogato, nel 1933, era una della mezza dozzina rimaste.

Santo Cambianica ha letteralmente salvato la sua cantina nello stesso modo in cui la Chiesa cattolica ha salvato metaforicamente i suoi parrocchiani: trasformando il comune vino da tavola in qualcosa di sacro, nel vino usato per la Messa.

Il fatto che la cantina fosse stata denominata come un santo cattolico e che Cambianica avesse forti legami con la chiesa ha reso la transizione logica da entrambe le parti, e quindi l'azienda vinse un accordo per continuare a produrre vino sacramentale durante il proibizionismo. (Molte aziende vinicole producevano già vino da vendere alle chiese e alle sinagoghe, e durante il Proibizionismo quella pratica andò in overdrive).
"La maggior parte degli altri marchi non erano spirituali; avevano nomi come Sunny Side o Sunny Slope", sottolinea Steve Riboli, il pronipote di Cambianica e ora vice presidente della San Antonio Winery.

San Antonio "era un'azienda basata sulla fede", afferma Riboli. "Letteralmente".

Cambianica cambiò rapidamente la sua attività per adattarsi alla situazione, di per sé una sorta di processo di trasformazione che divenne emblematico per l'azienda.

Prima del proibizionismo, San Antonio era una piccola azienda vinicola, che produceva circa 5.000 casse di vino rosso, il tipo di vino venduto "a misura di famiglia", o in forma di brocca, agli immigranti locali e alle cinque chiese della zona.

Quando il Proibizionismo finì, stava producendo 20.000 casse. Oggi, San Antonio Winery è il più grande fornitore di vino sacramentale nel paese.

Se il centro di Los Angeles era un luogo logico per costruire una cantina quasi un secolo fa, non è certo dove ti aspetteresti di trovarne uno adesso.

La cantina occupa tre blocchi di quello che nel 2012 è un paesaggio orizzontale in gran parte industriale.

Le impronte della Piccola Italia sono deboli, il colore spettrale del cemento: il piccolo mercato Lanza Bros, che continua a funzionare lungo la strada della cantina, e la cantina stessa, con le sue bandiere e l'ingresso attentamente mantenuto.

Oltre a quello? Non rimane molto dei bungalow e dei negozi, dei pastifici e dei pescivendoli che costituivano la fiorente comunità.

Ma oltrepassate la soglia della cantina nel vasto complesso: 100.000 piedi quadrati di showroom e ristorante, sale di degustazione e impianti di imbottigliamento, fermentazione, invecchiamento, cantine e magazzini, e troverete la storia ovunque guardiate.

È nelle foto in bianco e nero di Cambianica; di suo nipote Stefano Riboli, padre di Steve, che arrivò dall'Italia nel 1936 a 15 anni per aiutare a gestire I'azienda; della moglie di Stefano, Maddalena, una teenager guida di una famiglia italiana immigrata a Guasti, in Ontario, in California.

Puoi vedere la storia nelle file di bottiglie di vino e negli enormi barili di sequoia da quattro pollici di spessore, così grandi da poter contenere fino a 25.000 litri di vino, che popolano le stanze come i resti di una foresta di vecchia crescita.

San Antonio produce e imbottiglia la maggior parte delle oltre 500.000 casse di vino che produce annualmente in L.A.

Agli inizi degli anni '60, San Antonio era diventata I'ultima azienda vinicola rimasta in Los Angeles, e nel 1966 fu designata come uno dei punti di riferimento culturali della città.

Tutta la storia riempie le bottiglie del vino sacramentale che costituisce ancora il 15\% della produzione annuale di San Antonio. 
I gusti sono cambiati nel corso degli anni, come la Chiesa è cambiata (I'uso del vino d'altare nei servizi cattolici è cresciuto dopo il Concilio Vaticano II, negli anni '60) e così il suo clero e parrocchiani si sono abituati ai vini oltre le varietà utilizzate per le cerimonie religiose.

Oggi San Antonio produce sei vini sacramentali, di cui quattro un rosso, un rosato, un leggero Moscato e un'Angelica - sono i più popolari.

I vini aromatici una volta erano molto dolci e per la maggior parte fortificati - il diritto canonico stabilisce che il vino per l'Eucaristia deve essere "dal frutto della vite".

Riboli dice che la stragrande maggioranza del vino da messa ora è mediamente secca, e che i suoi vini non hanno aggiunta di acqua o zucchero.

Dall'inizio degli anni '90, sacerdoti e dirigenti parrocchiali hanno chiesto all'industria vini più secchi e colori più chiari.

Prima di allora, il vino sacramentale era scuro, apprezzato per il suo colore profondo che suggeriva il sangue di Cristo rappresentato.

Ma i vini più leggeri non solo hanno attirato i palati sia del clero che dei parrocchiani, hanno anche avuto un valore pragmatico - poiché i vini più leggeri sono più facili da pulire quando si rovesciano inevitabilmente, e quindi hanno meno probabilità di macchiare i panni dell'altare.

Immaginate le vostre bollette per il lavaggio a secco dopo una serata, ad esempio, di pot-au-feu e Cabernet Sauvignon.

La cattedrale di Nostra Signora degli Angeli nel centro di Los Angeles ha utilizzato esclusivamente i vini sacramentali di San Antonio fin dall'apertura e ha persino quattro diverse etichette San Antonio nel suo negozio di articoli da regalo.
Mons. Kevin Kostelnik, il parroco della cattedrale, dice che i parrocchiani comprano il vino non solo per bere, ma come souvenir.

Kostelnik dice che prima dell'apertura della cattedrale nel 2002, ha formato un comitato di degustazione di vini per scegliere i vini sacramentali.

Il comitato si è recato nella vicina San Antonio per una degustazione e alla fine ha deciso sul rosé della Comunione, che è l'unico vino che la cattedrale usa per l'Eucaristia.

Ed è molto gettonato: 25 casse al mese, oltre 300 bottiglie, ad un costo approssimativo di \$1.500. "San Antonio è un tesoro", dice Kostelnik. "Non ci sono molte aree urbane che dispongono di una cantina. Sono un modello di sacrificio per stare in città".

Per quanto riguarda il vino stesso, il monsignore afferma che la qualità del vino è importante sia per ragioni teologiche che estetiche.

"Non usiamo vino economico. È il sangue di Cristo: vogliamo usare il miglior vino che possiamo trovare".

Realizzato secondo il diritto canonico, San Antonio produce il suo gruppo di vini sacramentali da uve coltivate nella California settentrionale della Valle di San Joaquin da vitigni più vecchi con sapori intensi: il rosso è una miscela che comprende uve Barbera e Cabernet; il rosé è fatto con Grenache.

L'Angelica è fortificata con l'acquavite d'uva, come era consuetudine con le precedenti generazioni di vini d'altare, per ottenere il 18 percento di alcol.

Traduzione dal Smithsonian Magazine Institution del 6 dicembre 2017. Per saperne di più: www.smithsonianmag.com/travel/saved-fromprohibition-by-holy-wine-88250788 / \# fCD5XIUcQB4deAfI.99

\section{NOTIZIE IN BREVE}

\section{Pubblicato "FeDerSerD Informa n. 30 - gennaio 2018"}

La newsletter, in distribuzione e pubblicata sul sito www.federserd.it, è dedicata al:

“PROTOCOLLO OPERATIVO TRA FeDerSerD (Federazione Italiana degli Operatori dei Dipartimenti e dei Servizi delle Dipendenze) e CONAMS (Coordinamento Nazionale Magistrati di Sorveglianza) PER LA APPLICAZIONE DELL'AFFIDAMENTO IN PROVA IN CASI PARTICOLARI AI SENSI DELL'ART. 94 DEL DPR 309/90", recentemente presentato a Roma, presso il Senato della Repubblica.

Indice:

1. Premessa

2. Situazione attuale: analisi del contesto

3. Criticità

4. Finalità

5. Protocolli e linee guida di possibile riferimento

6. Azioni di miglioramento

6.1. certificazione stato tossico/alcoldipendenza

6.2. attualità della tossico/alcoldipendenza
6.3. analisi della strumentalità della richiesta

6.4. formulazione programma terapeutico (ambulatoriale, semi-residenziale, residenziale) e certificazione idoneità

6.5. monitoraggio programma terapeutico

7. Monitoraggio e revisioni del protocollo operativo

8. Bibliografia minima

9. Allegati:

A. fac simile "Certificazione stato di tossico/alcol dipendenza"

B. fac simile "Valutazione multidisciplinare"

C. fac simile "Certificazione idoneità programma terapeutico"

D. fac simile "Programma terapeutico Ambulatoriale"

D1. fac simile "Programma terapeutico residenziale/semiresidenziale"

E. fac simile "Monitoraggio programma terapeutico"

F. fac simile "Nota di trasmissione della documentazione"

Nel prossimo numero di MISSION verrà dato ampio spazio al fondamentale documento per la operatività dei Servizi. 